\title{
Josephson Current through a Superconducting Quantum Point Contact Shorter than the Coherence Length
}

\author{
C. W. J. Beenakker and $\mathrm{H}$. van Houten \\ Philips Research Laboratories, 5600 JA Eindhoven, The Netherlands
}

(Received 21 March 1991)

\begin{abstract}
It is shown theoretically that the critical current of a smooth and impurity-free superconducting constriction increases stepwise as a function of its width. The step height at zero temperature is $e \Delta_{0} / \hbar$, dependent on the energy gap $\Delta_{0}$ of the bulk superconductor but not on the junction parameters. This is the analog of the quantized conductance of a point contact in the normal state. It is essential that the constriction is short compared to the superconducting coherence length.
\end{abstract}

PACS numbers: $74.50 .+\mathrm{r}, 73.20 . \mathrm{Dx}, 85.25 . \mathrm{Cp}$

A few years ago it was discovered ${ }^{1,2}$ that the conductance of a ballistic point contact is quantized in units of $2 e^{2} / h .^{3}$ The origin of this phenomenon is the quantization of transverse momentum in the constriction. Each of the $N$ spin-degenerate transverse modes at the Fermi energy $E_{F}$ in such a quantum point contact contributes $2 e^{2} / h$ to the conductance. It is well known that the critical current of a tunnel junction or weak link is related to its normal-state conductance. ${ }^{4.5}$ What happens if the weak link is a quantum point contact? That question ${ }^{6}$ is addressed in this paper.

The case that the weak link is a classical ballistic point contact has been treated by Kulik and Omel'yanchuk. ${ }^{7}$ They obtained the current-phase-difference relationship $I(\delta \phi)$, and the critical current $I_{c} \equiv \max I(\delta \phi)$, for a point contact which is so much wider than the Fermi wavelength $\lambda_{F}$ that the quantization of transverse momentum can be ignored. Their analysis is based on a classical Boltzmann-type transport equation for the Green's functions (the Eilenberger equation ${ }^{5}$ ), and is not applicable to a quantum point contact.

The present analysis is based on the Bogoliubov-de Gennes (BdG) equation for quasiparticle wave functions, into which the Green's functions can be expanded. ${ }^{8} \mathrm{We}$ assume adiabatic transport through the constriction (no scattering between the modes), and treat the propagation of each mode using a WKB method. ${ }^{9}$ This level of description corresponds to that used by Glazman et al. ${ }^{10}$ in their treatment of the conductance quantization in the normal state. The adiabatic approximation requires that the width of the constriction varies slowly on the scale of $\lambda_{F}$, and hence that its length $2 L \gg \lambda_{F}$. In a superconductor a new length scale appears, the coherence length $\xi_{0} \equiv \hbar v_{F} / \pi \Delta_{0}$ [with $v_{F}$ the Fermi velocity and $\Delta_{0}(T)$ the temperature-dependent energy gap of the bulk superconductor]. Since $\Delta_{0}$ is smaller than $E_{F}$ by several orders of magnitude, one can have $\lambda_{F} \ll L \ll \xi_{0}$. As we will show, in the limit $L / \xi_{0} \rightarrow 0$ the discrete part of the excitation energy spectrum consists of an $N$-fold degenerate level at energy $\epsilon=\Delta_{0} \cos (\delta \phi / 2)$ [with $\delta \phi \in(-\pi, \pi)$ ]. Only the discrete spectrum contributes to the Josephson current in the WKB approximation. At $T=0$ we find $I(\delta \phi)$
$=N\left(e \Delta_{0} / \hbar\right) \sin (\delta \phi / 2)$, and hence $I_{c}=N e \Delta_{0} / \hbar$. In the classical limit $N \rightarrow \infty$, our results agree with Ref. 7 .

The critical current thus increases stepwise as the point contact is widened gradually. The step height $e \Delta_{0} / \hbar$ is independent of the parameters characterizing the Josephson junction. This remarkable feature originates from the insensitivity of the discrete spectrum to the properties of the junction for $L \ll \xi_{0}$. In this respect the bound states obtained here differ from the Andreev levels " in an SNS junction with $L_{N} \gg \xi_{0}(S$ denotes superconductor, $\mathrm{N}$ denotes normal metal, and $L_{N}$ is the separation of the two $\mathrm{SN}$ interfaces), where the discrete spectrum and critical current depend on $L_{N} .{ }^{12}$ Furusaki, Takayanagi, and Tsukada ${ }^{13}$ have very recently studied the Josephson current through a quantum point contact in the normal region of an SNS junction with $L_{N} \gg \xi_{0}$. These authors find that an adiabatic point contact, for which the conductance quantization is exact, ${ }^{10}$ nevertheless does not show steps in the critical current. They obtain a stepwise increasing $I_{c}$ for some nonadiabatic geometries, but the step height depends sensitively on the parameters of the junction. The condition $L \ll \xi_{0}$ of the present work is essential for a generic, junction-independent behavior.

Let us now consider an impurity-free superconducting constriction (Fig. 1), whose cross-sectional area $S(x)$ widens from $S_{\min }$ to $S_{\max } \gg S_{\min }$ over a length $L \gg \lambda_{F}$

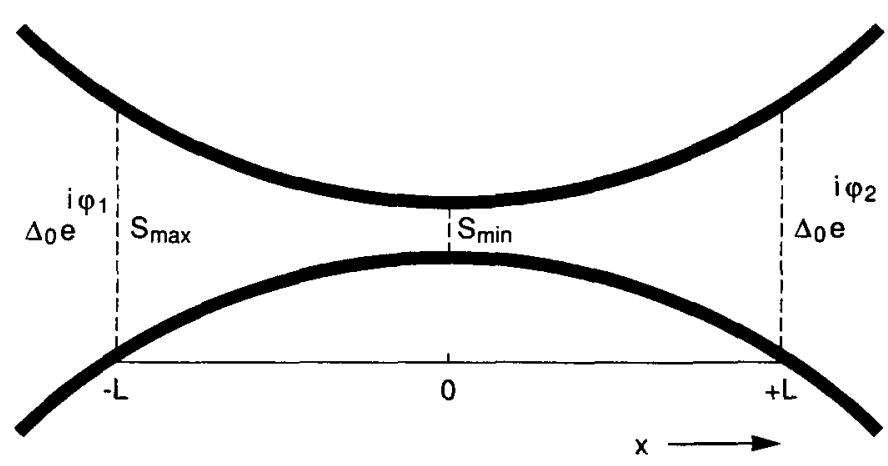

FIG. 1. Schematic drawing of a superconducting constriction of slowly varying width. 
and $\ll \xi_{0}$. The $x$ axis is along the constriction, and the area $S_{\max }$ is reached at $x= \pm L$. We are interested in the case that only a small number $N \simeq S_{\min } / \lambda_{F}^{2}$ of transverse modes can propagate through the constriction at energy $E_{F}$, and we assume that the propagation is adiabatic, i.e., without scattering between the modes.

In the superconducting reservoirs to the left and right of the constriction the pair potential (of absolute value $\Delta_{0}$ ) has phase $\phi_{1}$ and $\phi_{2}$, respectively. The characterization of the reservoirs by a constant phase is not strictly correct. The phase of the pair potential has a gradient in the bulk if a current flows. The gradient is $1 / \xi_{0}$ when the current density equals the critical current density in the bulk. In our case the critical current is limited by the point contact, so that the gradient of the phase in the bulk is much smaller than $1 / \xi_{0}$ lby a factor $S_{\min } / S(x)$ $\ll 1]$. Since the Josephson current is determined by the region within $\xi_{0}$ from the junction, one can safely neglect this gradient.

As we enter the constriction from, say, the left reservoir, the pair potential $\Delta(\mathbf{r})$ begins to vary from the bulk value $\Delta_{0} \exp \left(i \phi_{1}\right)$, for two reasons: (a) because the small number of transverse modes leads to a nonuniform density; and (b) because of contributions from the $N$ modes which have reached $\mathbf{r}$ from the right reservoir. Nonuniformities in $\Delta$ due to (a) and (b) are of order $1 / N(x)$ $\simeq \lambda_{F}^{2} / S(x)$ and $N / N(x) \simeq S_{\min } / S(x)$, respectively $[N(x)$ being the number of propagating modes at $x]$. For $|x|>L$ we have both $S(x) \gg \lambda_{F}^{2}$ and $S(x) \gg S_{\min }$. One may therefore neglect these nonuniformities for $|x|>L$, and put

$$
\Delta(\mathbf{r})= \begin{cases}\Delta_{0} e^{i \phi_{1}} \text { if } x<-L, \\ \Delta_{0} e^{i \phi_{2}} \text { if } x>L .\end{cases}
$$

Note the difference with planar SNS junctions, where $\Delta$ recovers its bulk value only at a distance $\xi_{0}$ from the SN interface. ${ }^{8}$. The much shorter decay length for nonuniformities due to a constriction is a geometrical "dilution" effect, well known in the theory of weak links. ${ }^{5,7}$ No specific assumptions are made on the variation of $\Delta$ in the narrow part of the constriction. In particular, our analysis also applies to a nonsuperconducting constriction $\left[\Delta(r)=0\right.$ at $\left.S_{\min }\right]$. Equation (1) remains valid up to terms of order $S_{\min } / S_{\max } \ll 1$.

We wish to calculate the current $I(\delta \phi)$ which flows in equilibrium through the constriction, for a given (time independent) phase difference $\delta \phi \equiv \phi_{1}-\phi_{2} \in(-\pi, \pi)$ between the two reservoirs. The equilibrium state is described by the eigenfunctions $\Psi \equiv(u, v)$ of the BdG equation $^{8}$

$$
\left(\begin{array}{cc}
\mathscr{H} & \Delta \\
\Delta^{*} & -\mathscr{H}
\end{array}\right) \Psi=\epsilon \Psi,
$$

where $\mathcal{H}=\mathrm{p}^{2} / 2 m+V(\mathrm{r})-E_{F}$ is the single-electron
Hamiltonian in a confining potential $V$. The energy $\epsilon$ is measured relative to $E_{F}$. The equilibrium current density $j(r)$ is obtained from the (normalized) eigenfunctions by

$\mathrm{j}=2 \frac{e \hbar}{m} \sum_{\epsilon>0} \operatorname{Re}\left\{f(\epsilon) u^{*} i \nabla u+[1-f(\epsilon)] v i \nabla_{v}^{*}\right\}$,

where the sum is over all states with positive eigenvalue, and $f(\epsilon)=\left[1+\exp \left(\epsilon / k_{B} T\right)\right]^{-1}$ is the Fermi function.

The transverse modes $|n\rangle \equiv \varphi_{n}(\mathbf{r})$ are eigenfunctions of $\left(p_{y}^{2}+p_{z}^{2}\right) / 2 m+V(\mathbf{r})$, with eigenvalues $E_{n}(x)$. We expand $\Psi(\mathbf{r})=\sum_{n}\left(u_{n}, v_{n}\right) \varphi_{n}$ into transverse modes and neglect off-diagonal matrix elements $\left\langle n|\mathscr{H}| n^{\prime}\right\rangle$ and $\left\langle n|\Delta| n^{\prime}\right\rangle(\langle\cdots\rangle$ denotes integration over $y$ and $z)$. This is the adiabatic approximation. The functions $u_{n}(x)$ and $v_{n}(x)$ then satisfy the one-dimensional $\mathrm{BdG}$ equation,

$$
\begin{aligned}
& \left(p_{x}^{2} / 2 m-U_{n}\right) u_{n}+\Delta_{n} v_{n}=\epsilon u_{n}, \\
& -\left(p_{x}^{2} / 2 m-U_{n}\right) v_{n}+\Delta_{n}^{*} u_{n}=\epsilon v_{n},
\end{aligned}
$$

where

$U_{n}(x)=E_{F}-E_{n}(x)-(1 / 2 m)\left\langle n\left|p_{x}^{2}\right| n\right\rangle \approx E_{F}-E_{n}(x)$,

and $\Delta_{n}(x)=\langle n|\Delta| n\rangle$ is the projection of $\Delta(\mathbf{r})$ onto the $n$th mode. We will consider one mode $n \leq N$ at a time, and omit the subscript $n$ for notational simplicity in most of the equations.

We use the WKB method of Bardeen et al., ${ }^{9}$ which consists of substituting

$$
\left(\begin{array}{l}
u \\
v
\end{array}\right)=\left(\begin{array}{c}
e^{i \eta / 2} \\
e^{-i \eta / 2}
\end{array}\right) \exp \left(i \int_{0}^{x} k\left(x^{\prime}\right) d x^{\prime}\right)
$$

into Eq. (4) and neglecting second-order derivatives (or products of first-order derivatives). The resulting equations for $\eta(x)$ and $k(x)$ are

$$
\begin{aligned}
& -\left(\hbar^{2} / 2 m\right) k \eta^{\prime}+\epsilon=|\Delta| \cos (\eta-\phi), \\
& \left(\hbar^{2} / 2 m\right)\left(k^{2}-i k^{\prime}\right)-U=i|\Delta| \sin (\eta-\phi),
\end{aligned}
$$

where $\Delta(x) \equiv|\Delta(x)| e^{i \phi(x)}$. In general, both $\eta$ and $k$ are complex. The WKB approximation requires that $U$ changes slowly on the scale of $\lambda_{F}$, so that reflections due to abrupt variations in the confining potential can be neglected. Reflections (accompanied by a change in sign of $\operatorname{Re} k$ ) due to spatial variations in the pair potential are negligible provided that $|\Delta|$ is much smaller than the kinetic energy $U$ of motion along the constriction. Since $U \gtrsim E_{F}-E_{N}(0)$, the WKB method cannot treat the threshold regime where $E_{F}$ lies within $\Delta_{0}$ of the cutoff energy $E_{N}(0)$ of the highest mode $N$ at the narrowest point of the constriction $(x=0)$. The energy separation $\delta E \equiv E_{N+1}(0)-E_{N}(0) \simeq E_{F} / N$ is much larger than $\Delta_{0}$ for small $N$, so that the threshold regime $\left|E_{F}-E_{N}(0)\right|$ $\leqslant \Delta_{0}$ consists only of small intervals in Fermi energy (smaller than the nonthreshold intervals by a factor of $\left.\Delta_{0} / \delta E \ll 1\right)$. 
For $|x|>L$, where $\Delta$ is independent of $x$, one has a constant $\eta$ which can take on the two values $\eta^{e}$ and $\eta^{h}$,

$$
\eta^{e, h}=\phi+\sigma^{e, h} \arccos \left(\epsilon / \Delta_{0}\right),
$$

where $\sigma^{e} \equiv 1, \sigma^{h} \equiv-1$. We have $\phi=\phi_{1}$ for $x<-L$ and $\phi=\phi_{2}$ for $x>L$. The function arccost is defined such that $\arccos t \in(0, \pi / 2)$ for $0<t<1$; for $t>1$, one has $i \arccos t=\ln \left[t+\left(t^{2}-1\right)^{1 / 2}\right]$. The WKB wave furctions $\Psi_{ \pm}^{e_{i} h}(x)$ for $|x|>L$ describe an electronlike $(e)$ or holelike $(h)$ quasiparticle with positive $(+)$ or negative $(-)$ wave vector,

$$
\begin{aligned}
& \Psi_{ \pm}^{e^{, h}}=\left(k^{e, h}\right)^{-1 / 2}\left(\begin{array}{c}
e^{i \eta^{e, h / 2}} \\
e^{-i \eta^{e, h / 2}}
\end{array}\right) \exp \left( \pm i \int_{0}^{x} k^{e, h} d x^{\prime}\right), \\
& k^{e, h}=\left(2 m / \hbar^{2}\right)^{1 / 2}\left[U+\sigma^{e, h}\left(\epsilon^{2}-\Delta_{0}^{2}\right)^{1 / 2}\right]^{1 / 2} .
\end{aligned}
$$

The square roots are to be taken such that $\operatorname{Re} k^{e, h} \geq 0$, $\operatorname{Im} k^{e} \geq 0, \operatorname{Im} k^{h} \leq 0$. The wave function (9) is a solution for $|x|>L$ of the BdG equation up to second-order derivatives.

For $\epsilon>\Delta_{0}$, the wave vectors $k^{e, h}$ are real at $|x|>L$, and hence the wave functions (9) remain properly bounded as $|x| \rightarrow \infty$. Since the states $\Psi_{+}^{e_{+}, h}$ and $\Psi_{-}^{e^{, h}}$ carry equal but opposite current, with the same density of states, there is no contribution to the Josephson current from the continuous spectrum. This is special for the WKB approximation, which assumes a smoothly varying $\Delta$. An abrupt variation in $\Delta$ may lead to Andreev reflections also for $\epsilon>\Delta_{0}$, and hence to part of the current being carried by the continuous spectrum. ${ }^{12}$

For $0<\epsilon<\Delta_{0}$, the two bounded WKB wave functions are

$$
\Psi_{+}=\left\{\begin{array}{l}
A_{+} \Psi_{+}^{h} \text { if } x<-L, \\
B_{+} \Psi_{+}^{e} \text { if } x>L,
\end{array}\right.
$$

while $\Psi$ - has the labels $e$ and $h$ interchanged (and subscripts - instead of + ). The transition from $k^{h}$ to $k^{e}$ on passing through the constriction (associated with a change in sign of $\operatorname{Im} k$ ) is analogous to Andreev reflection at an $\mathrm{SN}$ interface. " Andreev reflections are to be distinguished from ordinary reflections involving a change in sign of $\mathrm{Re} k$. Ordinary reflections due to the pair potential are neglected in the WKB approximation. By matching the wave functions $\Psi_{ \pm}$to the region $|x|<L$ we obtain a boundary-value problem with a discrete energy spectrum. Since $\epsilon<\Delta_{0} \ll U$, we may approximate $k \approx \pm\left(2 m U / \hbar^{2}\right)^{1 / 2}$ in Eq. (6) (the upper sign refers to $\Psi_{+}$, the lower sign to $\Psi_{-}$). The boundary-value problem then becomes

$$
\begin{aligned}
& \pm\left[\hbar^{2} U(x) / 2 m\right]^{1 / 2} \eta^{\prime}(x) \\
& +|\Delta(x)| \cos [\eta(x)-\phi(x)]=\epsilon, \\
& \eta(-L)=\phi_{1} \mp \arccos \left(\epsilon / \Delta_{0}\right), \\
& \eta(+L)=\phi_{2} \pm \arccos \left(\epsilon / \Delta_{0}\right) \text {. }
\end{aligned}
$$

Noting that $\eta$ is real, one deduces from Eq. (12) the in- equality $\left|\eta^{\prime}\right|<(\epsilon+|\Delta|)\left(\hbar^{2} U / 2 m\right)^{-1 / 2}$. Since $|\Delta| \lesssim \Delta_{0}$ and $U \gtrsim E_{F}-E_{N}(0)$, we have the limiting behavior

$$
|\eta(L)-\eta(-L)| \lesssim\left(L / \xi_{0}\right)\left[1-E_{N}(0) / E_{F}\right]^{-1 / 2} \rightarrow 0
$$

in the limit $L / \xi_{0} \rightarrow 0$. Hence, to order $L / \xi_{0}$ the boundstate energy $\epsilon$ is determined by

$$
\arccos \left(\epsilon / \Delta_{0}\right)= \pm \frac{1}{2} \delta \phi,
$$

independent of the precise behavior of $\Delta(\mathbf{r})$ in the constriction. Since $\arccos \left(\epsilon / \Delta_{0}\right)>0$, there is a single bound state per mode, with energy independent of the mode index $n \leq N$. The normalized WKB wave function is given by (using $\Delta_{0} \ll U$ and $\xi_{0} \gg L$ )

$$
\begin{aligned}
\Psi_{ \pm}= & \left(\frac{m}{2 \hbar^{2}}\right)^{1 / 2}\left(\Delta_{0}^{2}-\epsilon^{2}\right)^{1 / 4} k_{0}^{-1 / 2}\left(\begin{array}{c}
e^{i \bar{\phi} / 2} \\
e^{-i \bar{\phi} / 2}
\end{array}\right) \\
& x \exp \left( \pm i \int_{0}^{x} k\left(x^{\prime}\right) d x^{\prime}\right),
\end{aligned}
$$

where $\bar{\phi}=\left(\phi_{1}+\phi_{2}\right) / 2, k_{0}=\left(2 m U / \hbar^{2}\right)^{1 / 2}$, and

$$
k(x)=k_{0}(x)\left(1+\frac{i|\Delta(x)|}{2 U(x)} \sin [\bar{\phi}-\phi(x)]\right) .
$$

In accordance with Eq. (14), the wave function of the bound state is $\Psi_{+}$for $0<\delta \phi<\pi$ and $\Psi-$ for $-\pi<\delta \phi$ $<0$. The Josephson current $I_{n}$ due to mode $n \leq N$, evaluated in the constriction ${ }^{14}$ from Eqs. (3) and (15), is given by $I_{n}= \pm(e / \hbar)\left(\Delta_{0}^{2}-\epsilon^{2}\right)^{1 / 2}[1-2 f(\epsilon)]$. Substituting $\epsilon=\Delta_{0} \cos (\delta \phi / 2)$, and summing over the $N$ propagating modes, we obtain the total Josephson current

$$
I(\delta \phi)=N \frac{e}{\hbar} \Delta_{0}(T) \sin (\delta \phi / 2) \tanh \left(\frac{\Delta_{0}(T)}{2 k_{B} T} \cos (\delta \phi / 2)\right) .
$$

Since $N$ is an integer, Eq. (17) tells us that $I$ for a given value of $\delta \phi$ increases stepwise as a function of the width of the constriction. At $T=0$ we have $I(\delta \phi)$ $=N\left(e \Delta_{0} / \hbar\right) \sin (\delta \phi / 2)$, with a critical current $I_{c}=N e \Delta_{0} /$ $h$ (reached at $\delta \phi=\pi$ ). Near the critical temperature $T_{c}$ we have

$$
I(\delta \phi)=N\left(e \Delta_{0}^{2} / 4 \hbar k_{B} T_{c}\right) \sin (\delta \phi),
$$

and the critical current is reached at $\delta \phi=\pi / 2$. (The characteristic temperature is $T_{c}$ rather than $\delta E / k_{B}$, because of the condition $\Delta_{0} \ll \delta E$.) The ratio $I / G$ (with $G=2 N e^{2} / h$ the normal-state conductance of the quantum point contact) does not contain $N$ and is formally identical to the result for a classical point contact. ${ }^{7}$ We refer to Refs. 5 and 7 for graphs of the temperature dependence of $I(\delta \phi) / G$ and $I_{c} / G$.

Observation of the discretized critical current predicted in this paper requires the fabrication of a constriction with continuously variable width, or electron density. The narrow part of the constriction need not consist of a superconducting material. Quantum point contacts have 
been realized in the two-dimensional electron gas (2DEG) of a GaAs-(Al,Ga) As heterostructure, ${ }^{1-3}$ but it may be difficult to use such a constriction as a weak link between superconducting banks because of the formation of Schottky barriers. The 2DEG in the surface inversion layer of $p$-type InAs does not suffer from this drawback. ${ }^{15}$ A negatively biased split-gate (insulated from the inversion layer) might be used to laterally deplete the 2DEG, in order to create a smooth and short constriction of variable width, connecting two superconducting banks. A problem with this S-2DEG-S structure is the large conduction-band offset at the 2DEG-S interface, which can induce spurious reflections that may complicate the observation of the effect.

In summary, we have presented the analog for the stationary Josephson effect of the conductance quantization in the normal state. We have focused on the case of an adiabatic constriction with a vanishingly small threshold regime. Future work will consider the effects of nonadiabaticity and the threshold behavior in the intervals $\left|E_{F}-E_{N}\right| \lesssim \Delta_{0}$. Observation of the discretization of the Josephson current predicted here presents an experimental challenge.

The authors acknowledge the stimulating support of M. F. H. Schuurmans.

'B. J. van Wees, H. van Houten, C. W. J. Beenakker, J. G. Williamson, L. P. Kouwenhoven, D. van der Marel, and C. T. Foxon, Phys. Rev. Lett. 60, 848 (1988).

${ }^{2}$ D. A. Wharam, T. J. Thornton, R. Newbury, M. Pepper, H. Ahmed, J. E. F. Frost, D. G. Hasko, D. C. Peacock, D. A. Ritchie, and G. A. C. Jones, J. Phys. C 21, L209 (1988).

${ }^{3} A$ review is given by $C$. W. J. Beenakker and $H$. van Houten, in Solid State Physics, edited by H. Ehrenreich and D. Turnbull (Academic, New York, 1991), Vol. 44, p. 1.

${ }^{4}$ V. Ambegaokar and A. Baratoff, Phys. Rev. Lett. 10, 486 (1963); 11, 104(E) (1963).

${ }^{5} \mathrm{~A}$ review of superconducting weak links is given by $\mathrm{K}$. $\mathrm{K}$. Likharev, Rev. Mod. Phys. 51, 101 (1979).
${ }^{6}$ H. van Houten, Appl. Phys. Lett. 58, 1326 (1991).

${ }^{7}$ I. O. Kulik and A. N. Omel'yanchuk, Fiz. Nisk. Temp. 3, 945 (1977); 4, 296 (1978) [Sov. J. Low Temp. Phys. 3, 459 (1977); 4, 142 (1978)].

${ }^{8}$ P. G. de Gennes, Superconductivity of Metals and Alloys (Benjamin, New York, 1966).

9J. Bardeen, R. Kümmel, A. E. Jacobs, and L. Tewordt, Phys. Rev. 187, 556 (1969).

${ }^{10}$ L. I. Glazman, G. B. Lesovik, D. E. Khmel'nitskii, and R. I. Shekhter, Pis'ma Zh. Eksp. Teor. Fiz. 48, 218 (1988) [JETP Lett. 48, 238 (1988)]; see also A. Yacoby and Y. Imry, Phys. Rev. B 41, 5341 (1990).

"IA. F. Andreev, Zh. Eksp. Teor. Fiz. 46, 1823 (1964); 49, 655 (1965) [Sov. Phys. JETP 19, 1228 (1964); 22, 455 (1966)].

12I. O. Kulik, Zh. Eksp. Teor. Fiz. 57, 1745 (1969) ISov. Phys. JETP 30, 944 (1970)]; C. Ishii, Prog. Theor. Phys. 44, 1525 (1970); J. Bardeen and J. L. Johnson, Phys. Rev. B 5, 72 (1972); A. V. Svidzinsky, T. N. Antsygina, and E. N. Bratus', J. Low Temp. Phys. 10, 131 (1973); G. B. Arnold, J. Low Temp. Phys. 59, 143 (1985); M. Büttiker and T. M. Klapwijk, Phys. Rev. B 33, 5114 (1986); V. Z. Kresin, Phys. Rev. B 34, 7587 (1986); A. Furusaki and M. Tsukada, Physica (Amsterdam) $165 \& 166$ B, 967 (1990); B. J. van Wees, K.-M. H. Lenssen, and C. J. P. M. Harmans (to be published).

${ }^{13}$ A. Furusaki, H. Takayanagi, and M. Tsukada (to be published).

${ }^{14}$ As remarked earlier, we have disregarded the gradient in the phase of the pair potential in the bulk reservoirs. This gradient $\left(\ll 1 / \xi_{0}\right)$ is required for current conservation in the bulk, but can be neglected if the current is calculated within the constriction $\left(|x| \lesssim L \ll \xi_{0}\right)$, cf. Refs. 5 and 7 . An alternative method of calculating $I(\delta \phi)$, which gives the same results as the method used here, is to apply the relation $I=(-2 e)$ $\hbar) d F / d \delta \phi$ between the Josephson current and the derivative of the free energy $F$ with respect to the phase difference. For a description of this alternative calculation, see, C. W. J. Beenakker and $\mathrm{H}$. van Houten, in Proceedings of the International Symposium on Nanostructures and Mesoscopic Systems, Santa Fe, 20-24 May 1991 (to be published).

${ }^{15} \mathrm{H}$. Takayanagi and T. Kawakami, Phys. Rev. Lett. 54, 2449 (1985). 